\title{
Amphiphilic nanotubes in the crystal structure of a biosurfactant protein hydrophobin HFBII
}

\author{
Johanna M. Kallio, ${ }^{* a, b}$ Juha Rouvinen ${ }^{a}$ \\ Received (in $X X X, X X X$ ) Xth $X X X X X X X X X 200 X$, Accepted Xth $X X X X X X X X X 200 X$ \\ First published on the web Xth $X X X X X X X X X 200 X$ \\ DOI: $10.1039 / \mathbf{b 0 0 0 0 0 0 x}$
}

\begin{abstract}
Atomic scale experimental data by X-ray crystallography has been collected on an amphiphilic protein nanotube, consisting of a biosurfactant protein Trichoderma reesei hydrophobin HFBII.
\end{abstract}

Hydrophobins are small, amphiphilic proteins with an innate ability to self-assemble in a hydrophobin-hydrophilic interface. Hydrophobins are secreted by filamentous fungi, and the ability to self-assemble at air-water interface serves in is lowering the surface tension of water during hyphal growth while assembly on the cell wall - air interface forms a protective coating on the fungal surface when growing into the air ${ }^{1}$. The amphiphilicity of the assemblies and the molecule itself arises from a patch of hydrophobic residues on 20 the protein surface ${ }^{2}$, even though proteins typically have the majority of hydrophobic residues in the core of the protein The assembled structures of hydrophobins are patterned and well-ordered, which opens way to nanotechnological applications $^{3}$. Spontaneous self-assembly of hydrophobins has 25 been shown to reverse the hydrophobicity of a surface, once brought in contact with a hydrophobic solid (such as Teflon) or a hydrophilic surface ${ }^{1}$.

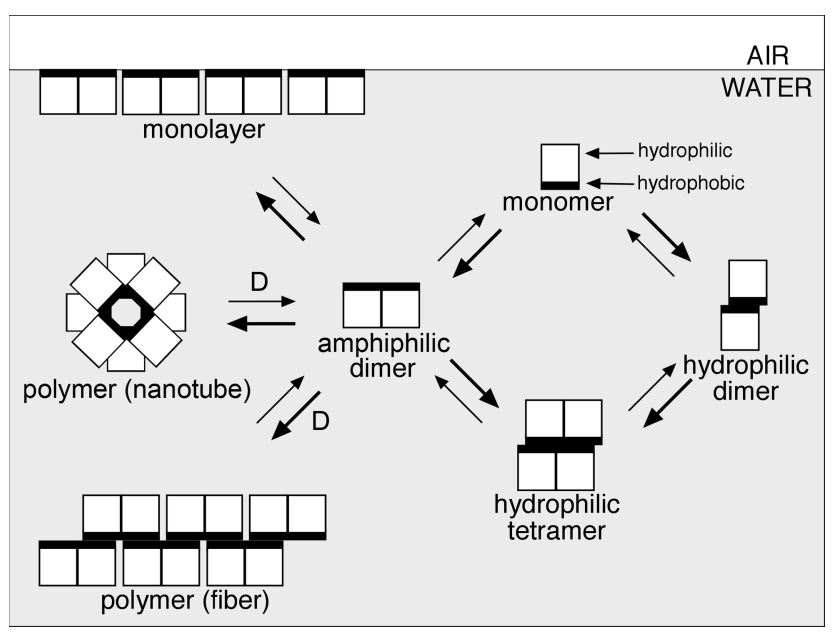

Scheme 1. Functional mechanism of hydrophobins based on ${ }_{30}$ self-assembly. The fiber structures are formed via detergent (D) interactions.

Our previous crystallographic studies of Trichoderma reesei hydrophobins HFBI and HFBII have shown that the controlled 35 assembly of hydrophobins extends far beyond the formation of an amphiphilic film on a hydrophobic-hydrophilic interface $^{2}$ and allowed us to draft the scheme of functional mechanism in atomic scale. Depending on - or controlled by the conditions, different ways of molecular packing may be 40 obtained while the conformation of the monomers remains the same. In this work we describe a yet new type of assembly of HFBII, obtained when crystallized in the presence of polystyrene nanospheres ${ }^{4}$.

The fold of the protein molecules, as also observed 45 previously, composes of four antiparallel $\beta$-strands and an $\alpha$ helix. The $\beta$-strands form a small barrel, which is reinforced by two disulfide bridges. Two additional disulfide bridges join the $\alpha$-helix and the $\mathrm{N}$-terminal loop to the $\beta$-barrel. Very modest signs of changes, mainly in the side chain so conformations, were observed in the protein molecule in comparison to the previous crystallographic structures of the same protein, excluding the residues Asp59 and Glu60 for which both the main and the side chains were in distinct conformations.

55 The two molecules in the asymmetric unit, designated as molecules A and B (Figure 1), lie side-by-side in such away that the hydrophobic surface areas (composed of residues Leu7, Val18, Leu19, Leu21, Ile22, Val24, Val54, Ala55, Va157, Ala58, Ala61, Leu62, Leu63 in HFBII) are aligned in 60 uniform direction. This arrangement is similar to the amphiphilic dimer described in the previous fibrilar sturucture 2PL7, with the distinction that the detergent molecule is crammed between the two protein molecules instead of lying on top of the hydrophobic surface (Supplementary ${ }_{65}$ information).

While no detergents were directly added to the crystallization solution, the nanosphere suspension contained residual amounts of sodium dodecyl sulphate (SDS) alike detergent, likely to help to solubilize the polystyrene 70 nanospheres. When a large, linear electron density was detected in the vicinity of hydrophobic surface areas (Supplementray information), partially buried by the protein molecules, SDS was refined to the residual density. The hydrophobic tail of the SDS interacts with the side chains of 75 surrounding hydrophobic residues (Val7 and Phe8 from molecules $\mathrm{A}$ and $\mathrm{B}$ ) and the oxygens of the sulphate group form hydrogen bonds to the main chain nitrogens (Val7, Phe8) in molecule B.

Due to detergent intervention, no uniform hydrophobic 80 surface is formed by the adjacent protein molecules. However, takig into account the hydrophobic aromatic residue Phe 8 and the hydrophobic tail of the detergent, a uniform hydrophobic surface is formed in combination of protein molecules and the detergent. The nature of the detergent seems to affect the 


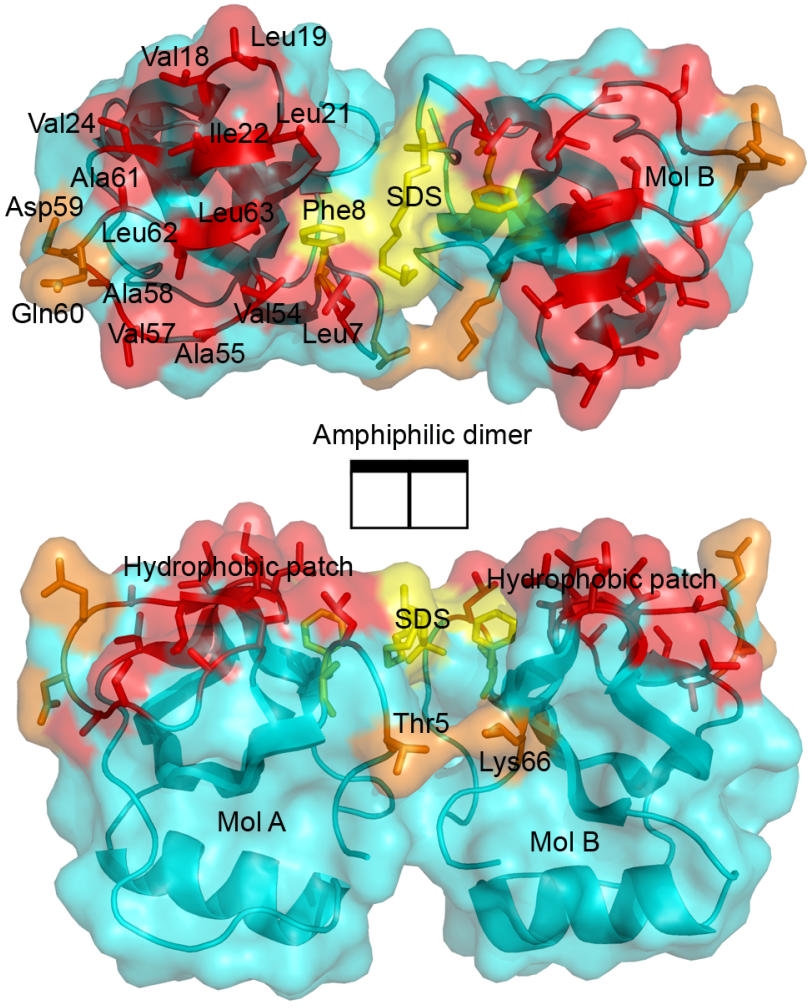

Figure 1. The asymmetric unit of hydrophobin HFBII in space group I222 with cartoon and surface representations. The hydrophobic surfaces are in red and labeled for molecule A in top panel. The SDS and Phe 8 contributing to the 5 continuity of the hydrophobic surface area are in yellow and Asp59 and Gln with conformational change are in orange. Above, the asymmetric unit viewed perpendicular to hydrophobic surface areas and below the side view.

10 coordination to the hydrophobic parts of the protein and thus effecting the entire self-assembly process. A smaller hydrophilic head group, such as the sulphate in SDS or the dimethylamine-oxide in LDAO, allows the detergent molecule to be more incorporated into the packing of the protein 15 molecules, whereas a larger sugar group, such as the glucose moiety in heptyl- $\beta$-D-thioglucoside (HSG) and octyl- $\beta$-Dthioglucoside (OSG), allows the coordination of the hydrophobic tail only (Supplementry information). In addition to interactions mediated by the detergent, there is a hydrogen 20 bond between the molecules of the asymmetric unit form molecule A Thr5 OG1 to molecule B Lys66 NZ. The rest of the interacting residues are from the $\mathrm{N}$ - and $\mathrm{C}$-termini.

The basic unit of the amphiphilic tubes is an octamer, composed of four molecules A and four molecules B (Figure ${ }_{25} 2 \mathrm{a}$ ). Within the octameric array, in addition to interaction between the molecules in the asymmetric unit, each molecule $\mathrm{A}$ is in contact with two symmetry related molecules B, and vice versa. Here, the hydrogen bonding interaction is between Gln60 NE2 and Thr16 OG1 and residues Val18, Leu19,

${ }_{30}$ Leu21, Ile22, Val54, Ala55, Val57, Ala61, Leu62, and Leu63 from the hydrophobic patch of both molecules contribute to the interactions. The formation of this important hydrogen bond for octamer interaction is the likely cause for distinct confromations of the residues Asp59-Gln60 in comparion to
35 previous HFBII structures. Gln60 is also an outlier in the Ramachandran plot in spite of unambiguous electron density for the residue.

Adjacent octamers packing side by side in uniform orientation create the tube-like arrangement (Figure 2b). A 40 salt bridge between molecule B Asp25 OD2 and molecule A Lys49 NZ in the adjacent octamers is mediating the contacts. The tube-like arrays extend through the crystal and adjacent tubes are in contact by interactions in the helical region (Figure 2c). However, no hydrogen bonds are formed between 45 the adjacent tubes. The most important interactions within and between the octamers are summarized in Supplementray information.

A small tunnel, below $10 \AA$ in diameter, is left inside the protein octamer. At the inner surface of the array is located an ${ }_{50}$ abundance of the residues of the hydrophobic surface area, creating an amphiphlic nature for the tubes. The diameter of one hydrophobin monomer is slightly less than $30 \AA$ and the diameter of the tube is approximately $60 \AA$. Large solvent channels are left between the outern, or the hydrophilic, walls 55 of the individual tubes. These solvent channels are about $50 \AA$ in diameter. The solvent content of these crystals is about $61 \%$, which is in the range typical for protein crystals.

The interaction between the two protein molecules of the aymmetric unit is exactly the same as an interaction found ${ }_{60}$ between adjacent molecules in the previously determined structure (Supplementary information, structure 2PL6), which hints that the formation of this structure is not random but a result of the sophisticated mechanism by which this protein self-assembles. It is remarkable, that the basic building block ${ }_{65}$ i.e. the hydrophobin molecule can produce such divers oligomeric structures yet the molecule itself is rather rigid. However, the fold of the protein allows some plasticity to enhance interactions and to adopt to a new environment ${ }^{2}$.

Much effort has been directed in producing protein or 70 peptide nanotubes by using a computational approach, template-synthesis or protein engineering ${ }^{5}$ Other nanostructures (micelles, vesicles, ribbons, fibers and tubes) consisting solely of biomaterials also exist, as the discovery of carbon nanotubes has pushed towards fabrication of organic 75 and inorganic nanostructures. Self-assembled micro- and nanotubes of phospholipids, glycolipids, bolaamphiphiles and two-component systems have been developed ${ }^{5}$. Also a DNA single-strand has been rationally designed to form nanotubes by self-assembly ${ }^{5}$.

${ }_{80}$ Artificial self-assembled nanotubes of almost complete protein, hydrolyzed milk protein $\alpha$-lactalbumin, has been described $^{5}$, with potential applications in food and non-food industry. Nanotubes may also be formed by self-assembly of surfactant-like peptides ${ }^{6}$, in which case the driving force in ${ }_{85}$ self-assembly is to bury the hydrophobic tails of the peptides. What we present here is a case of a natural, intact surfactant protein producing a hollow nanotube structure that is amphiphilic itself.

\section{Notes and references}

\footnotetext{
$90{ }^{a}$ Department of Chemistry, University of Eastern Finland, PO Box 111, 80101 Joensuu, Finland.
} 
${ }^{b}$ EMBL Hamburg c/o DESY, Notkestraße 85, 22603 Hamburg, Germany. Fax: +49 4089902 149; Tel: +49 4089902 176; E-mail:

johanna.kallio@embl-hamburg.de

* The protein material was produced and purified at the VTT Technical 5 Research Center as previously described ${ }^{7}$. The lyophilized protein material was dissolved in pure water to $8 \mathrm{mg} / \mathrm{ml}$. The crystals grew from solution of $10 \%$ polyethylene glycol (MW 2000), $0.2 \mathrm{M}$ lithium sulphate and $0.1 \mathrm{M}$ Tris-buffer at $\mathrm{pH} 8.5$ using the vapour-diffusion method with handing drops. The crystallization droplet contained $50 \%$ protein 10 solution, $40 \%$ of precipitant solution and $10 \%$ of Nanosphere Size Standard suspension purchased from the Duke Scientific Corporation. The nanosphered were $50 \mathrm{~nm} \pm 2.0 \mathrm{~nm}$ in size and their density in aqueous suspension is $1.05 \mathrm{~g} / \mathrm{cm} 3$.

The data collection were collected at the EMBL Hamburg 5 Outstation/DESY at beamline $\mathrm{X} 12$ to $1.9 \AA$ resolution. Unit cell parameters: $\mathrm{a}=42.052 \AA, \mathrm{b}=91.359 \AA, \mathrm{c}=94.808 \AA$. Space group I222. Data collection $1.000 \AA$ wavelength. Resolution limits at the highest resolution shell were $2.2-1.9 \AA$ and in data collection statistics the numbers in parentheses refer to this highest resolution shell. Number of 20 observations was 101637 (33898) and number of unique reflections was 27376 (9524). Completeness $98.4 \%$ (96.4\%). $\mathrm{R}_{\text {meas }} 8.5 \%$ (32.5\%). I / $\sigma$ (I) $12.72(4.91)$.

The data were process with the $\mathrm{XDS}^{8}$ program and refined in Phenix ${ }^{8}$. Final $\mathrm{R}=21.38 \%, \mathrm{R}_{\text {free }}=26.33 \%$. RMSD bond length $0.007 \AA$ and

${ }_{25} \mathrm{RMSD}$ bond angle $\quad 1.227^{\circ}$. Number of protein atom, water molecules, and other atoms in the final model were 972,92 , and 32, respectively. The average B-factor was $24 \AA^{2}$

Pseudomerohedral twinnig was detected in the data in $\mathrm{Xtriage}^{8}$ and the structure was refined using twin law $-h,-l,-k$. The twin fraction was 0.340

30 usin proportional detwin mode. The program $\operatorname{Coot}^{6}$ was used to evaluate the electron density maps. The structure has been deposited to the Protein Data Bank (3QQT). PISA ${ }^{8}$ was used to evaluate the interfaces and assemblages.

1 H.A.B. Wösten, F.H.J .Schuren, J.G.H. Wessels, EMBO J., 1994, 13, 5848-5854; J.G.H. Wessels, Trends Plant Sci., 1996, 1, 9-14; M. Linder, Curr. Opin. Colloid Interface Sci., 2009 14, 356-363.

2 J. Hakanpää, A. Paananen, S. Askolin, T. Nakari-Setälä, T. Parkkinen, M. Penttilä, M.B. Linder, J. Rouvinen, J. Biol. Chem., 2004, 279, 534-539; J. Hakanpää, M. Linder, A Popov, A. Schmidt, J. Rouvinen, Acta Cryst., 2006 D62 356367; J. Hakanpää, G.R. Szilvay, H. Kaljunen, M. Maksimainen, M. Linder, J. Rouvinen Protein Sci., 2006, 15, 2129-2140; J.M. Kallio, M.B. Linder, J. Rouvinen, J. Biol. Chem. 2007, 282, 28733-28739.

3 K. Kurppa, H. Jiang, G.R. Szilvay, A.G. Nasibulin, E.I Kauppinen, M.B. Linder, Angew. Chem. Int. Edit., 2007, 46 , 6446-6449; P. Laaksonen, J. Kivioja, A. Paananen, M. Kainlauri, K. Kontturi, J. Ahopelto, M.B. Linder, LangmuirI, 2009, 25, 5185-5192; H. Valo, P. Laaksonen, L. Peltonen, M.B. Linder, J.T. Hirvonen, T.J. Laaksonen, ACS Nano, 2010, 4, 1750-1758

4 J.M. Kallio, N. Hakulinen, J.P. Kallio, M.H. Niemi, S Kärkkäinen, J. Rouvinen, PLoS One, 2009, 4, e4198. F. Hodzhaoglu, F. Kurniawan, V. Mirsky, C. Nanev, Cryst. Res. Technol., 2008, 43, 588-593.

5 H.Y. Lee, S.R. Nam, J.I. Hong, Chem Asian J., 2009, 4, 226235; H. Liu, Y. Chen, Y. Hu, A.E. Ribbe, C. Mao Angew Chem. Int. Ed., 2006, 45, 1942-1945; C.J. Tsai, J. Zheng, R. Nussinov, PLoS Comput. Biol., 2006, 2, e42; S. Hou, J. Wang, C.R. Martin, Nano Lett., 2005, 5, 231-234; G.F. Audette, E.J. van Schqaik, B. Hazes, R.T. Irvin, Nano Lett., 2004, 4, $1897-$ 1902; M.T. Kumara, N. Srividya, S. Muralidharan, B.C. Tripp, Nano Lett., 2006, 6, 2121-2129.

6 J.F. Graveland-Bikker, C.G. de Kruif, Trends in Food Sci. Technol., 2006, 17, 196-203; S. Vauthey, S. Santoso, H. Gong, N. Watson, S. Zhang, Proc. Natl. Acad. Sci., 2002, 99, 53555360

7 T. Nakari-Setälä, N. Aro, M. Ilmen, G. Munos, N. Kalkkinen, M. Penttilä, Eur. J. Biochem., 1997, 248, 415-423.
8 W. Kabsch, J. Appl. Crystallogr., 1993, 26, 795-800; P.D. Adams, P.V. Afonine, G. Bunkóczi, V.B. Chen, I.W. Davis, N. Echols, J.J. Headd, L.W. Hung, G.J. Kapral, R.W. GrosseKunstleve, A.J. McCoy, N.W. Moriarty, R. Oeffner, R.J. Read, D.C. Richardson, J.S. Richardson, T.C. Terwilliger, P.H. Zwart, Acta Cryst., 2010 D66, 213-221; P. Emsley, K. Cowtan Acta Cryst., 2004, D60, 2126-2132; E. Krissinel, K. Henrick, J. Mol. Biol., 2007, 372, 774-707.
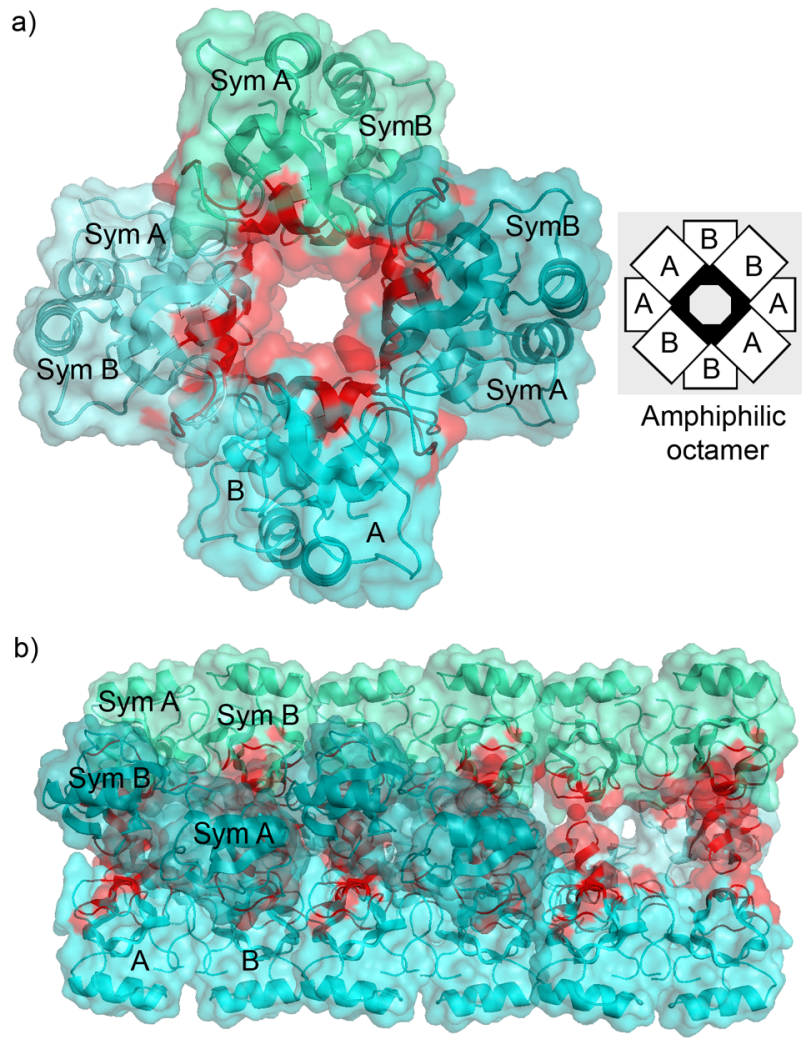

c)
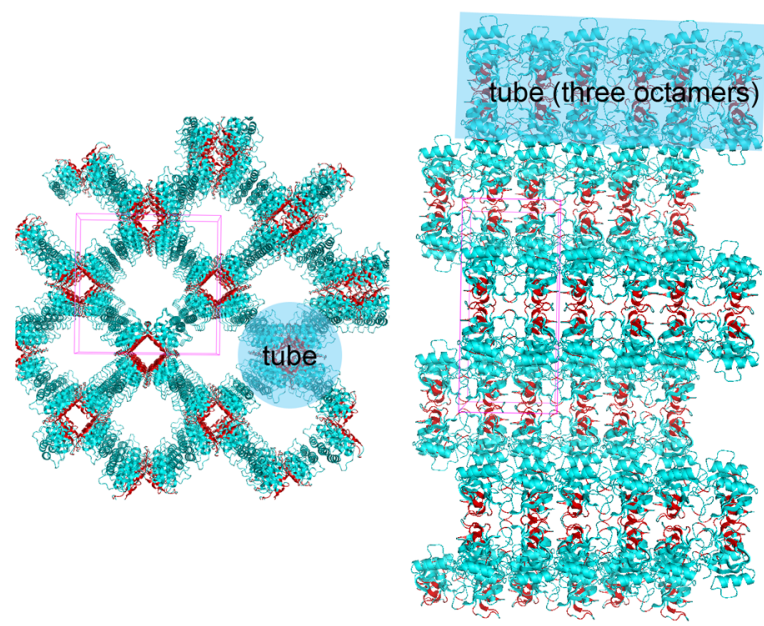

Figure 2. The formation of crystal structure and the nanotube 80 array: a) four asymmetric units combine to an octamer b) a tube structure is formed of consecutive octamers (one dimer omitted from the right to show the interior) c) crystal structure is formed of nanotubes packing side-by-side. The hydrophobic surface areas in red and each asymmetric unit in a) and b) are 85 colored with different shade for clarity. Amphiphilic tube, formed of octamers is highlighted in c) both in direction of aaxis and b-axes of the crystal. 